\title{
Empyema in children hospitalised at Chris Hani Baragwanath Academic Hospital, Johannesburg, South Africa: A retrospective study
}

\author{
A Ghoor, ${ }^{1}$ MMed (Paed); T Mabaso, ${ }^{1}$ MMed (Paed); K Mopeli, ${ }^{1}$ MMed (Paed); A Izu, ${ }^{2,3}$ PhD; S A Madhi, ${ }^{2,3}$ PhD; S G Lala, ${ }^{1} \mathrm{PhD}$; \\ C Verwey, ${ }^{1,2,3}$ MMed (Paed); Z Dangor, ${ }^{1,2,3} \mathrm{PhD}$ \\ ${ }^{1}$ Department of Paediatrics and Child Health, Faculty of Health Sciences, University of the Witwatersrand, Johannesburg, South Africa \\ ${ }^{2}$ South African Medical Research Council Respiratory and Meningeal Pathogens Research Unit, Faculty of Health Sciences, University of the \\ Witwatersrand, Johannesburg, South Africa \\ ${ }^{3}$ National Research Foundation/Department of Science and Technology: Vaccine Preventable Diseases, Faculty of Health Sciences, University of \\ the Witwatersrand, Johannesburg, South Africa
}

Corresponding author: Z Dangor (ziyaad.dangor@wits.ac.za)

\begin{abstract}
Background. There is a paucity of information on empyema in children from low- and middle-income countries since the introduction of the pneumococcal conjugate vaccine.

Objectives. To describe the aetiology and management of empyema in a setting of high HIV and tuberculosis (TB) prevalence.

Methods. A retrospective descriptive study was undertaken between January 2012 and December 2016 in children aged $<14$ years at a large secondary-tertiary referral hospital in Soweto, South Africa. Cases of empyema were identified through administrative databases. Clinical, laboratory and radiological data were extracted from patient records.

Results. We identified 65 cases of protocol-defined empyema, including 22 (33.8\%) referred from surrounding hospitals. The median age at presentation was 53.2 months (interquartile range (IQR) 19.5 - 103.6). Thirteen patients (20.0\%) were HIV-infected and 6 (9.2\%) were HIVexposed but uninfected. A bacterial pathogen was identified in 36 cases (55.3\%). The commonest causative organisms were Staphylococcus aureus $(14 / 65,21.5 \%)$ and Streptococcus pneumoniae (5/65, 7.7\%). Treatment for TB, initiated in 28 children (43.1\%), was more frequent in HIV-infected children $(10 / 13,76.9 \%)(p=0.011)$; however, microbiological evidence of TB was present in only 5 cases $(7.7 \%)$. Forty-three children $(66.2 \%)$ had an intercostal drain (ICD) inserted and $16(24.6 \%)$ a pigtail percutaneous catheter, while a fibrinolytic was only used in $6(10.2 \%)$. Eight children $(12.3 \%)$ had a thoracotomy and $7(10.7 \%)$ had video-assisted thorascopic drainage, all of whom had a prior ICD inserted, a median of 20 days (IQR 10 - 33) before surgery. Overall, 7 children (10.8\%) were mechanically ventilated and 1 (1.5\%) died. Conclusions. Our study showed a dominance of $S$. aureus as a cause of empyema. A high proportion of HIV-infected children with empyema were initiated on TB treatment, highlighting challenges in managing TB-HIV co-infection. Although fibrinolytics or early surgery are recommended, neither practice was common in this setting.
\end{abstract}

S Afr Med J 2018;108(12):1055-1058. DOI:10.7196/SAMJ.2018.v108i12.13099

The incidence of empyema has reportedly increased in some highincome countries since the introduction of childhood seven-valent pneumococcal conjugate vaccine (PCV), ${ }^{[1-4]}$ although there is a paucity of such data from low- and middle-income settings. Recent studies from South Africa (SA) and the USA suggested a decrease in the incidence of empyema since the introduction of 13 -valent PCV. ${ }^{[5,6]}$ Nonetheless, Streptococcus pneumoniae (serotypes 1, 3 and 19A) remains the commonest (10 - 48\%) pathogen identified, followed by Staphylococcus aureus (8 - 19\%), and less commonly Haemophilus influenzae and Mycoplasma pneumoniae. ${ }^{[3,5]}$ In tuberculosis (TB)endemic settings, Mycobacterium tuberculosis (MTB) may account for $10-14 \%$ of cases. ${ }^{[5]}$ However, studies in high-income countries indicate that in most instances the aetiology of the empyema may be unknown, as $<30 \%$ of blood, pleural fluid or sputum cultures yield an organism. ${ }^{[3]}$ The use of molecular techniques has been shown to improve diagnostic yield. ${ }^{[4]}$

The management of empyema remains challenging, even in highincome countries. ${ }^{[3]}$ Antibiotics alone have been recommended in children with no respiratory compromise, and fibrinolytics are recommended with either pigtail or intercostal drain (ICD) insertion in patients with respiratory compromise or failure to respond to antibiotics. Early video-assisted thorascopic surgery (VATS) for drainage, used as first-line treatment, has not been shown to improve outcomes at 6 months compared with use of an ICD and fibrinolytics. ${ }^{[7,8]}$ The use of fibrinolytics may reduce the need for more invasive and expensive surgical options.

\section{Objectives}

To describe the clinical epidemiology of empyema at a secondarytertiary hospital with a paediatric pulmonology referral service, in a setting of high HIV and TB prevalence.

\section{Methods}

A retrospective descriptive study was undertaken in children aged $<14$ years at Chris Hani Baragwanath Academic Hospital (CHBAH) over the 5-year period January 2012 - December 2016. CHBAH is a secondary-tertiary academic hospital situated in Soweto, a periurban and mostly low-income township south of Johannesburg, SA. In addition to providing a paediatric service to the residents of Soweto, this hospital is the referral centre for hospitals in the surrounding areas. The prevalence of childhood HIV infection is 3 $4 \%$, while $30 \%$ of newborns are HIV-exposed in this setting. ${ }^{[9]}$ 
The paediatric pulmonology department at $\mathrm{CHBAH}$ was established in February 2011. Children admitted or referred from other hospitals with empyema are admitted to the general paediatric wards and management includes referral to the pulmonologist. Doctors diagnosed empyema by visualising purulent material drained from the pleural space or on ultrasound and/or a computed tomography (CT) scan. There was no standardised treatment protocol for the management of empyema; however, all children with empyema receive broad-spectrum antibiotics (ampicillin and gentamicin, or co-amoxiclav). Other treatment modalities include an ICD, a pigtail drain, fibrinolytics, VATS or thoracotomy with drainage/cleanout.

Children diagnosed with empyema at $\mathrm{CHBAH}$ over the past 5 years were identified through the pulmonology and discharge summary administrative databases. Clinical, laboratory and radiological information was extracted from patient records. Laboratory markers such as a full blood count, C-reactive protein, blood cultures, sputum cultures, fluid biochemistry and culture were obtained from the National Health Laboratory Service Track-Care system. Sputum or gastric washings were routinely cultured for MTB but not for other micro-organisms.

\section{Statistical analysis}

Strengthening the Reporting of Observational Studies in Epidemiology (STROBE) criteria were used for the inclusion of cases that met the case definition. Empyema was defined as the visualising of purulent/pus/turbid fluid after thoracocentesis or drainage; an ultrasound scan of the chest demonstrating fibrin strands, loculations or dense fluid; a CT scan demonstrating hyperdense fluid or splitting of the pleura; a neutrophil predominance $(\geq 2+)$ on pleural fluid microscopy; or a bacterial organism identified on culture of the pleural fluid. The incidence was calculated for patients who presented directly to CHBAH (patients not resident in Soweto were excluded in the incidence calculation), and expressed as cases per 100000 population for Soweto and surrounding areas as per Statistics South Africa population estimates (Gauteng subdistrict population midyear estimates 2005 - 2016: personal communication from Mrs Andy Valashiya, Directorate: Information Management, National Department of Health, 13 May 2013). Probable causative pathogens were identified using pleural, blood or sputum cultures, or molecular techniques in two cases.

Data were described using the following statistical methods: the median and interquartile range (IQR) were reported for continuous variables and frequencies were reported for categorical variables. The Mann-Whitney test was used to compare continuous variables and Pearson's $\chi^{2}$ test or Fisher's exact test for categorical variables. Data were analysed using Stata version 13.1 (StataCorp, USA).

The study was approved by the University of the Witwatersrand Human Research Ethics Committee (ref. no. M170152).

\section{Results}

\section{Demographic details}

Over the 5-year period, 65 of the 154 screened database cases met the case definition of empyema (Fig. 1). Overall, 37 patients (56.9\%) were male and the median age at diagnosis was 53.2 months (IQR 19.5 103.6) (Table 1). Thirteen children (20.0\%) were HIV-infected and $6(9.1 \%)$ were aged $<18$ months and HIV-exposed but uninfected. The median weight-for-age $z$-score was -1.3 (IQR $-2.1--0.2$ ) and the median height-for-age $z$-score was -1.6 (IQR $-2.7-0.8$ ). The incidence of empyema was estimated as 1.46 ( $95 \%$ confidence interval (CI) 1.05 - 1.97) per 100000 population and 3.40 (95\% CI 2.45 - 4.59) per 1000 hospitalised cases of acute lower respiratory infection, and

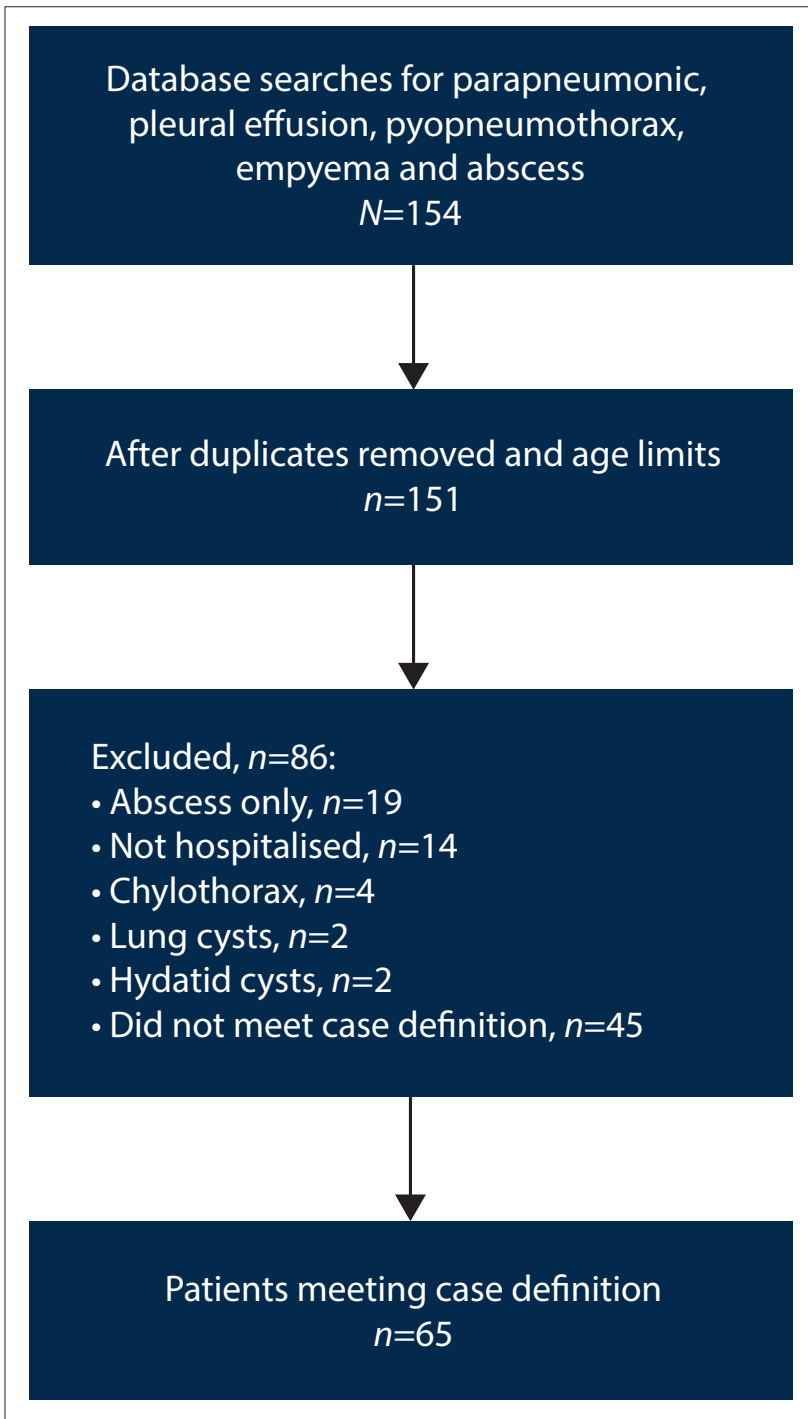

Fig. 1. Flow diagram of cases meeting case definition.

did not differ significantly over the study period $(p=0.19)$. A third of the patients $(22,33.8 \%)$ were referred from surrounding hospitals, with a median time to referral of 14 days (IQR 9 - 19).

\section{Aetiological agents}

A bacterial pathogen was identified in 36 cases (55.3\%), 34 on culture of blood or pleural fluid and 2 isolated by multiplex polymerase chain reaction (Fig. 2). The most common organism isolated was Staphylococcus aureus $(n=14,21.5 \%)$ followed by Streptococcus pneumoniae $(n=5,7.7 \%), \operatorname{MTB}(n=5,7.7 \%)$ and Klebsiella pneumoniae $(n=3,4.6 \%)$. One patient (1.5\%) grew both MTB and S. aureus on pleural fluid, while the other 4 cases of MTB were cultured on gastric washings or sputum samples.

Because 28 children (43.1\%) were treated for TB, in an exploratory analysis we compared clinical characteristics between those treated for $\mathrm{TB}$ and those not treated (Table 1). Treatment for TB was initiated more commonly in HIV-infected $(10 / 13,76.9 \%)$ than HIVuninfected children $(17 / 51,33.3 \%)(p=0.011)$.

\section{Laboratory findings}

The median white cell count was $19.8 \times 10^{9} / \mathrm{L}(\mathrm{IQR} 13.6-27.3)$ (Table 2). Forty-five patients $(69.2 \%)$ had neutrophilia, with a median neutrophil count of $13.5 \times 10^{9} / \mathrm{L}$ (IQR $7.3-19.2$ ). Although 
Table 1. Demographic characteristics of children with empyema

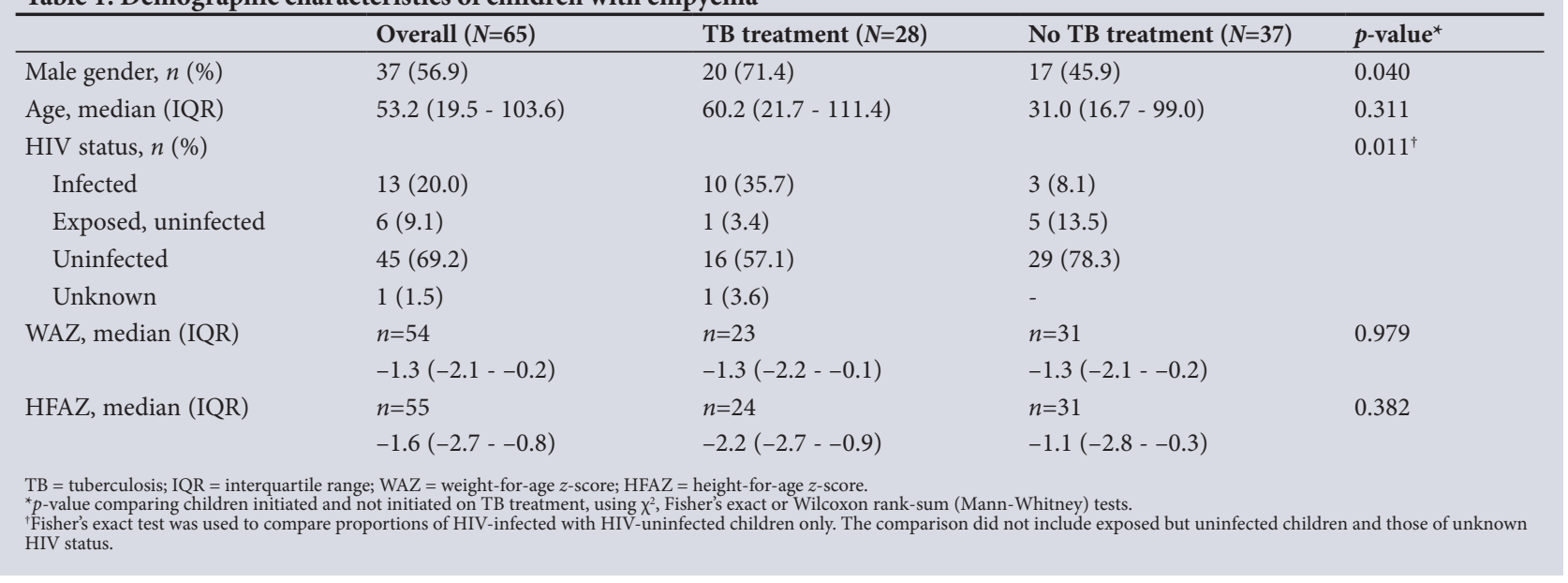

53 patients $(81.5 \%)$ had monocytosis, no significant difference was noted between those who were treated for $\mathrm{TB}$ and those who were not. The C-reactive protein level was raised in all cases, with a median of 143 mg/L (IQR 71 - 294). Children who were treated for TB had a significantly higher pleural fluid protein level $(58 \mathrm{~g} / \mathrm{L})$ than those who were not $(50 \mathrm{~g} / \mathrm{L}) \quad(p=0.030)$, whereas pleural fluid lactate dehydrogenase was lower in those who were treated for TB (965 U/L v. $2243 \mathrm{U} / \mathrm{L})(p=0.004)$.

Treatment modalities and outcomes The median duration of hospitalisation was 18 days (IQR 12 - 24). All children were initiated on antibiotic therapy and 59 (90.8\%) required pleural drainage. Fortythree children (66.2\%) had an ICD inserted and $16(24.6 \%)$ had a pigtail percutaneous catheter inserted; however, fibrinolytics were only documented in 6 (9.2\%). Eight children (12.3\%) had a thoracotomy and 7 (10.7\%) had VATS, all of whom had a prior drain inserted with a median of 20 days (IQR 10 33) from admission to surgery. Seven children $(10.8 \%)$ required intubation and ventilation and 1 died (case fatality ratio $1.5 \%$ ).

\section{Discussion}

In this low- to middle-income setting, the commonest causes of empyema were S. aureus, S. pneumoniae and MTB. The lower prevalence of $S$. pneumoniae empyema may be attributable in part to the introduction and high coverage rates (>99\%) of the 13-valent PCV since 2011 in this setting. ${ }^{[5,6,10]}$ However, our study was limited because we relied mainly on cultureconfirmed diagnosis, and studies using molecular techniques continue to show a higher prevalence of $S$. pneumoniae as the predominant pathogen causing empyema. ${ }^{[4]}$ Importantly, antibiotics initiated in children

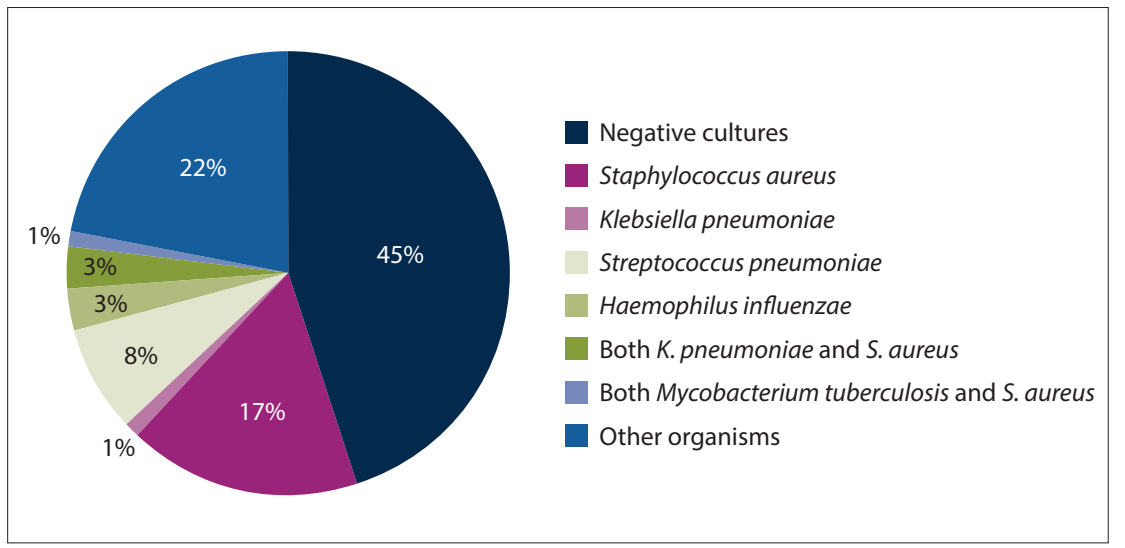

Fig. 2. Bacterial pathogens identified on blood or pleural fluid. 'Other organisms' identified on pleural fluid ( $\mathrm{n}=10$ patients) were Acinetobacter species and Serratia marcescens ( $\mathrm{n}=1$ ), Enterococcus faecalis $(\mathrm{n}=1)$, E. faecium $(\mathrm{n}=1)$, streptococcal species $(\mathrm{n}=1)$, Ochrobactrum anthropi and Acinetobacter baumannii $(\mathrm{n}=1)$, Kluvera intermedia $(\mathrm{n}=1)$, Streptococcus anginosus $(\mathrm{n}=1)$, Citrobacter koserii $(\mathrm{n}=1)$, Morganella morganii $(\mathrm{n}=1)$, and normal respiratory flora $(\mathrm{n}=1)$. On blood culture $(\mathrm{n}=4$ patients), Salmonella $\operatorname{spp} .(\mathrm{n}=1)$, S. viridans $(\mathrm{n}=2)$ and Corynebacterium $(\mathrm{n}=1)$ were identified.

with empyema should provide $S$. aureus and S. pneumoniae cover.

Another important finding of our study was the large proportion of patients who were initiated on TB therapy despite only a few being microbiologically confirmed. Most children have paucibacillary TB, and culture sensitivity is generally lower than in adults. ${ }^{[1,12]}$ Diagnosing TB is challenging in children, and clinicians often rely on clinical symptoms, radiological evidence and immunological testing, none of which are specific. ${ }^{[1]]}$ This is further compounded in HIV-infected children because of an overlap between clinical and radiological evidence of TB and other opportunistic or chronic infections. ${ }^{[13]}$ This could in part explain our findings where most HIVinfected children were initiated on $\mathrm{TB}$ treatment. A study in Cape Town, SA, where the burden of $\mathrm{TB}$ is among the highest in the world, reported a prevalence of TB empyema of $10-14 \%{ }^{[5]}$ Furthermore, culture-confirmed TB has been reported in $8-43 \%$ of children with acute pneumonia, and co-infection with other pathogens may occur in a quarter of children. ${ }^{[12,14]}$ In settings with a high TB burden, concurrent diagnostic evaluation for MTB as well as other organisms is therefore necessary in children with empyema.

The management of empyema includes fluid drainage if there is no clinical response to antibiotic therapy, or in cases of respiratory compromise. ${ }^{[3]}$ In our study, although the majority of cases required drainage, only a few received fibrinolytic therapy. This is in contrast to high-income settings, where the use of fibrinolytics is recommended with intercostal drainage, as the outcome is similar to that of VATS..$^{[7,8,15]}$ Furthermore, use of fibrinolytic therapy in low-resource settings may have additional value because the shortage of surgeons and postoperative intensive care facilities delays surgical intervention. 
Table 2. Laboratory characteristics of children with empyema

\begin{tabular}{|c|c|c|c|c|}
\hline & Overall & TB treatment & No TB treatment & $p$-value ${ }^{*}$ \\
\hline \multirow[t]{2}{*}{ Leucocytes $\left(\times 10^{9} / \mathrm{L}\right)$, median (IQR) } & $n=64$ & $n=27$ & $n=37$ & 0.176 \\
\hline & $19.8(13.6-27.3)$ & $19.0(9.1-23.1)$ & $20.0(15.9-28.0)$ & \\
\hline \multirow[t]{2}{*}{ Neutrophils $\left(\times 10^{9} / \mathrm{L}\right)$, median (IQR) } & $n=61$ & $n=25$ & $n=36$ & 0.268 \\
\hline & $13.5(7.3-19.2)$ & $10.4(7.3-17.2)$ & $14.2(7.5-23.1)$ & \\
\hline \multirow[t]{2}{*}{ Lymphocytes $\left(\times 10^{9} / \mathrm{L}\right)$, median (IQR) } & $n=61$ & $n=25$ & $n=36$ & 0.692 \\
\hline & $3.3(2.0-5.3)$ & $3.2(1.8-5.1)$ & $3.5(2.1-5.7)$ & \\
\hline \multirow[t]{2}{*}{ Monocytes $\left(\times 10^{9} / \mathrm{L}\right)$, median $(\mathrm{IQR})$} & $n=61$ & $n=25$ & $n=36$ & 0.450 \\
\hline & $1.6(0.9-2.5)$ & $1.5(0.9-2.3)$ & $1.6(1.1-2.7)$ & \\
\hline \multirow[t]{2}{*}{ CRP $(\mathrm{mg} / \mathrm{L})$, median (IQR) } & $n=49$ & $n=19$ & $n=30$ & 0.862 \\
\hline & $143(71-294)$ & $97(71-273)$ & $162(64-309)$ & \\
\hline \multirow[t]{2}{*}{ Pleural fluid protein $(\mathrm{g} / \mathrm{L})$, median (IQR) } & $n=29$ & $n=9$ & $n=20$ & 0.030 \\
\hline & $51(46-58)$ & $58(53-68)$ & $50(44-53)$ & \\
\hline \multirow[t]{2}{*}{ Pleural fluid LDH (U/L), median (IQR) } & $n=30$ & $n=10$ & $n=20$ & 0.004 \\
\hline & $2000(1321-2902)$ & $965(289-2000)$ & $2243(2000-3618)$ & \\
\hline
\end{tabular}

\section{Study limitations}

Our study had several limitations, including incomplete or missing records. We therefore used a strict case definition for empyema and are likely to have underestimated the incidence in this setting. Despite the high culture positivity rate (55.4\%), a large proportion (21.5\%) of organisms isolated from culture were probable contaminants. A major limitation was the lack of access to molecular testing to improve diagnostic yield. Furthermore, as no protocol for the management of empyema existed in our setting, investigations and management, including those related to $\mathrm{TB}$, were at the discretion of the treating clinician. Additionally, the high rates of TB therapy in our study must be taken in the context of delayed referral and suboptimal management of empyema, possibly biasing clinicians to initiate therapy based on delayed recovery. A comparison between patients receiving PCV7 and those who received PCV13 could not be made, as the vaccination information was unavailable. We did not have accurate data on the duration and choice of antibiotics or long-term outcome data.

\section{Conclusions}

This study shows that $S$. aureus, S. pneumoniae and MTB were the commonest causes of empyema. Furthermore, in settings with a high burden of TB or HIV, thorough investigations for concomitant TB infection are necessary in children presenting with empyema.

\section{Declaration. None.}

Acknowledgements. We are thankful to all the parents and children who participated in the study, Respiratory and Meningeal Pathogens Research Unit staff, and the registrars and consultants in the Department of Paediatrics at Chris Hani Baragwanath Academic Hospital.

Author contributions. Conception or design of the work: AG, CV and $\mathrm{ZD}$; acquisition, analysis or interpretation of data: AG, TM, KM, AI, SAM, SGL, CV and ZD; drafting the manuscript: AG and ZD; revising the manuscript critically: AG, TM, KM, AI, SAM, SGL, CV and ZD; final approval of the version to be published: AG, TM, KM, AI, SAM, SGL, CV and ZD.

Funding. SAM is funded in part by the Department of Science and Technology/National Research Foundation: South African Research Chair Initiative in Vaccine Preventable Diseases and the Medical Research
Council of South Africa. The funders had no role in study design, data collection and analysis, decision to publish, or preparation of the manuscript.

Conflicts of interest. None.

1. Roxburgh CS, Youngson GG, Townend JA, Turner SW. Trends in pneumonia and empyema in Scottish children in the past 25 years. Arch Dis Child 2008;93(4):316-318. https://doi.org/10.1136/ adc. 2007.126540

2. Grijalva CG, Nuorti JP, Zhu Y, Griffin MR. Increasing incidence of empyema complicating childhood community-acquired pneumonia in the United States. Clin Infect Dis 2010;50(6):805813. https://doi.org/10.1086\%2F650573

3. Walker W, Wheeler R, Legg J. Update on the causes, investigation and management of empyema in 3. Walker W, Wheeler R, Legg J. Update on the causes, investigation and management of em
childhood. Arch Dis Child 2011;96(5):482-488. https://doi.org/10.1136/adc.2009.165357

4. Zampoli M, Chaya S, Zar HJ. Empyema in children: Update of aetiology, diagnosis and management 4. Zampoli M, Chaya S, Zar HJ. Empyema in children: Update of aetiology, diagnosis and manage
approaches. Curr Pulmonol Rep 2017;6(1):16-25. https://doi.org/10.1007/s13665-017-0161-4

5. Zampoli M, Kappos A, Wolter N, et al. Etiology and incidence of pleural empyema in South African children. Pediatr Infect Dis J 2015;34(12):1305-1310. https://doi.org/10.1097/INF. 000000000000088

6. Simonsen L, Taylor RJ, Schuck-Paim C, Lustig R, Haber M, Klugman KP. Effect of 13-valent pneumococcal conjugate vaccine on admissions to hospital 2 years after its introduction in the USA: A time series analysis. Lancet Respir Med 2014;2(5):387-394. https://doi.org/10.1016/S22132600(14)70032-3

7. Sonnappa S, Cohen G, Owens CM, et al. Comparison of urokinase and video-assisted thoracoscopic surgery for treatment of childhood empyema. Am J Respir Crit Care Med 2006;174(2):221-227. https://doi.org/10.1164/rccm.200601-027OC

8. Marhuenda C, Barcelo C, Fuentes I, et al. Urokinase versus VATS for treatment of empyema: A randomized multicenter clinical trial. Pediatrics 2014;134(5):e1301-e1307. https://doi.org/ 10.1542/peds.2013-3935

9. National Department of Health, South Africa. The National Antenatal Sentinel HIV \& Syphilis National Department of Health, South Africa. The National Antenatal Sentinel HIV \& Syphilis
Prevalence Survey, South Africa, 2011. Pretoria: NDoH, 2012. www.hst.org.za/publications/ Prevalence Survey, South Africa, 2011. Pretoria: NDoH, 2012. www.hst.org.za/publications/
NonHST\%20Publications/Antenatal_Sentinel_survey_Report2012_final.pdf (accessed 24 October 2018)

10. Madhi SA, Bamford L, Ngcobo N. Effectiveness of pneumococcal conjugate vaccine and rotavirus vaccine introduction into the South African public immunisation programme. S Afr Med J 2014;104(3 Suppl 1):228-234. https://doi.org/10.7196/SAMJ.7597

1. Perez-Velez CM, Marais BJ. Tuberculosis in children. N Engl J Med 2012;367(4):348-361. https:// doi.org/10.1056/NEJMra1008049

12. Dube FS, Kaba M, Robberts FJ, et al. Respiratory microbes present in the nasopharynx of children hospitalised with suspected pulmonary tuberculosis in Cape Town, South Africa. BMC Infect Dis 2016;16(1):597. https://doi.org/10.1186/s12879-016-1934-z

13. Pitcher RD, Beningfield SJ, Zar HJ. The chest X-ray features of chronic respiratory disease in HIVinfected children - a review. Paediatr Respir Rev 2015;16(4):258-266. https://doi.org/10.1016/j. prrv.2015.01.005

14. Jeena PM, Pillay P, Pillay T, Coovadia HM. Impact of HIV-1 co-infection on presentation and hospital-related mortality in children with culture proven pulmonary tuberculosis in Durban, South Africa. Int J Tuberc Lung Dis 2002;6(8):672-678. http://www.ingentaconnect.com/content/ iuatld/ijtld/2002/00000006/00000008/art00007;jsessionid=1sdlv8xooq7bq.X-ic-live-01\# (accessed 2 November 2018)

15. Zampoli M, Kappos A, Verwey C, Mamathuba R, Zar HJ. Impact of fibrinolytics on the outcome of empyema in South African children. S Afr Med J 2015;105(7):549-553. https://doi.org/10.7196/ SAMJnew.7796

Accepted 17 May 2018 